\title{
Effects of Handgrip Exercise on the Shoulder Muscle Activation and Cross-Sectional Area of the Supraspinatus Muscle in Rotator Cuff Repair Patient
}

\author{
Dong-Rour Lee, $\mathrm{PT}, \mathrm{PhD} \cdot$ Young-Eun Choi, PT, $\mathrm{PhD}^{1 \dagger}$ \\ Department of Physical Rehabilitation Team, Good Gang-An Hospital \\ ${ }^{1}$ Department of Physical Therapy, College of Health Medicine, Kaya University
}

Received: January 6, 2020 / Revised: January 7, 2020 / Accepted: January 26, 2020

(C) 2020 J Korean Soc Phys Med

\begin{abstract}
| Abstract |
PURPOSE: This study examined the effects of handgrip exercise, which was started two weeks after surgery for shoulder rotator cuff repair, on the extent of muscle activation around the shoulder and the cross-sectional area of the supraspinatus muscle.
\end{abstract}

METHODS: Among patients diagnosed with rotator cuff rupture by an orthopedic surgeon and rotator cuff repair was performed using an arthroscope, 28 were selected as subjects. These subjects were allocated randomly to the experimental group and control group with 14 subjects in each group. An electromyogram was measured as a measure of the extent of muscle activation around the shoulder for a total of six times (\%RVC). The cross-sectional area of the supraspinatus muscle was measured before and after the rotator cuff repair by magnetic resonance imaging.

RESULTS: The extent of muscle activation in accordance

$\uparrow$ Corresponding Author : Young-Eun Choi

choiye00@naver.com, https://orcid.org/0000-0002-1419-8519

This is an Open Access article distributed under the terms of the Creative Commons Attribution Non-Commercial License (http://creativecommons.org/licenses/by-nc/3.0) which permits unrestricted non-commercial use, distribution, and reproduction in any medium, provided the original work is properly cited. with time in both the experimental group and control group displayed significant differences in various muscles including the anterior deltoid, pectoralis major, upper trapezius and infraspinatus muscle $(\mathrm{p}<.05)$. A significant difference in the variation of the cross-sectional area of the supraspinatus muscle was observed between the experimental group and the control group $(\mathrm{p}<.05)$.

CONCLUSION: Handgrip exercise helps rehabilitate the shoulder joint at the acute stage after rotator cuff repair when assertive exercise therapy cannot be applied.

Key Words: Handgrip exercise, Rotator cuff repair, Supraspinatus, Muscle cross-sectional area

\section{Introduction}

With the recent increase in the average lifespan and the participation of people in leisure life and sports activities, the number of patients complaining of shoulder joint pain is increasing [1]. Meyer and Gerber [2] asserted that gradual degenerative changes occur in the shoulder joint with time due to the repetitive actions of the joint, while Hong [3] reported that there was a decrease in cell count and deformation of articular surface due to the gradual degradation in the arrangement orderliness of the rotator 
cuff fibers as one ages. Treatments of rotator cuff damage are divided into conservative therapy and surgical therapy. Conservative therapies include drug therapy and physiotherapy for the alleviation of pain. Surgical therapy is required when such conservative therapies are ineffective in reducing pain.

Rotator cuff repair is affected by a range of factors, including the size of rupture, time of surgery, age of the patient, and muscular strength before surgery [4]. A high level of success in the outcome of surgical therapy on full thickness rotator cuff ruptures, in the range of $85 \sim 90 \%$, has been reported [5]. The importance of shoulder rehabilitation following arthroscopic reconstruction is emphasized increasingly with the presentation of numerous studies on the guidelines for the physiotherapy following arthroscopic reconstruction. Boudreau et al. [6] reported that the following exercises need to be implemented following the arthroscopic reconstruction of a rotator cuff rupture: isometric exercise for the rapid reduction of edema, exercise for the range of motion of a joint for the prevention of articular capsule contracture, stabilization exercise, proprioception training, rehabilitation of the neuromuscular system, muscular fortification exercise, and functional exercise. Currently, many surgeons are recommending the commencement of exercise as early as possible to prevent muscular atrophy and stiffening of the joint after rotator cuff repair [7].

Muscular atrophy, which occurs due to the fixation for the prevention of re-rupturing after rotator cuff repair, is considered an essential issue in rehabilitation following the suturing of the tendon. Psatha et al. [8] reported that the thickness of the muscle displayed the largest reduction up to the $15^{\text {th }}$ day after fixation due to the 43-day plaster cast period after suturing the Achilles tendon in the leg with no change in muscle thickness after the $30^{\text {th }}$ day. Therefore, limiting exercise of the shoulder joint for four weeks for the prevention of the re-rupturing following rotator cuff repair will induce muscle atrophy around the shoulder joint and harm the rehabilitation of the shoulder joint. To ensure the safe and efficient movement of the human body, the stabilization of the proximal joint needs to be preceded before movement of the distal joint. That is, efficient biodynamic movement that minimizes the load on the joint is possible when the structure involved in the proximal stability is activated first when all the movements of the human body are generated [9]. Therefore, stability of the shoulder joint, the proximal joint, is necessary for movement of the distal joint. Hence, handgrip exercise will bring about the activation of the rotator cuff, which plays the most crucial role in the stability of the shoulder. The handgrip can alter the muscle activity of the arm. Au and Keir [10] reported that gripping an object with a 30\% force led to a $7 \sim 10 \%$ decrease in the deltoid muscle activity compared to gripping with the maximum force. In addition, May et al. [11] showed that the handgrip is strongly associated with the activation of the rotator cuff by redistributing the force from the deltoid muscle and rotator cuff. Therefore, this study examined the effects of handgrip exercise during the acute phase after rotator cuff repair when active exercise therapy could not be applied, on shoulder joint pain, joint range of motion, muscle activity, shoulder function, and cross-sectional area of the supraspinatus muscle in patients with mild and moderate injuries.

\section{Methods}

\section{Study subjects}

Patients who visited the plastic surgery department of the Hospital G, Busan, Korea were selected as the subjects of this study. In particular, among the patients who visited the hospital with shoulder pain and were diagnosed with rotator cuff rupture by plastic surgeons, patients for whom rotator cuff repair had been performed using an arthroscope were selected as subjects. Thirty-three patients, who understood the intent of this study and consented voluntarily 
to participate in the study, were allocated randomly into two groups(17 in the control group and 16 in the experimental group). Five of these patients dropped out in the middle of the study: two due to a return to work and three due to rejection of the therapy. Finally, 28 subjects, 14 in the experimental group and 14 in the control group, participated in the study until the end of the experiment.

\section{Experimental procedures}

\section{1) Surface electromyogram measurement}

A surface EMG device(TeleMyo 2400T DTS, Noraxon, USA) was used to measure the extent of muscle activation around the shoulder joint. The sampling rate of the EMG signal was set to $1,000 \mathrm{~Hz}$. A frequency range of $20 \sim 450$ $\mathrm{Hz}$ was used with a notch filter set to $60 \mathrm{~Hz}$. To minimize resistance, the body hairs were removed, and the areas of attachment of the electrodes on the skin were cleaned thoroughly using an alcoholic cotton ball prior to the attachment of the $\mathrm{Ag} / \mathrm{AgCl}$ surface electrodes. After the protective film on the electrode was detached, the surface of the electrode covered with an electrolyte was attached in parallel to the running direction of the muscles at the center of the corresponding muscle by maintaining a distance of approximately $2 \mathrm{~cm}$ between the electrodes. Measurements were made on the following six muscles: the anterior deltoid, middle deltoid, posterior deltoid, pectoralis major, upper trapezius, and infraspinatus muscles of the injured shoulder joint. The locations of the electrodes were decided in accordance with the guidelines reported by Cram [12]. In this study, as an experiment implemented immediately after rotator cuff repair, the extent of muscle activation was measured by reference voluntary contraction (\%RVC) because the maximum isometric contraction could not be performed due to the risk of the re-rupture in the research subjects. To obtain the \%RVC for measuring the muscle activity around the shoulder, the RMS of the standard motion was first measured followed by the RMS of a certain motion. The subjects were instructed to assume a comfortably seated posture with the legs spread naturally to the width of the shoulder, with the soles of the feet touching the floor, pushing the hip to the deep end of the chair, and straightening the back for the experiment. The subjects, after the shoulder abduction brace had been removed, were then instructed to grasp the dynamometer naturally with a weight of $.7 \mathrm{~kg}$ and maintain the hold for five seconds while having the shoulder bent by $30^{\circ}$ in the direction of the scapular surface and bending the elbow joint by $90^{\circ}$. The data were computed using the EMG signals for three seconds by excluding those in the first second and the last second. This was repeated three times, with the average value used in further analysis. The subjects were allowed to take 60 seconds of rest between each of the measurements.

\section{2) Magnetic resonance image measurement}

A MR unit was used to examine the cross-sectional area of the supraspinatus muscle. The MR system(1.5T Signa Excite, GE, USA) was used to take images by setting the device to the T1 weighted image, TR 450, TE 16, spin echo and a resolution of $320 \times 224$. The atrophy of supraspinatus muscle was measured at the location, at which the $\mathrm{Y}$ shaped structure made by the scapular spine and coracoacromion, and at the tip of clavicle among the oblique sagittal planes of the $\mathrm{T} 1$ weighted image in accordance with the measurement method reported by Thomazeau et al. [13]. Software was used, and the cross-sectional area of the muscles was obtained using a tracing method.

\section{3) Intervention}

In this study, the experiment was conducted by dividing the subjects into the control group, for which six weeks of general physiotherapy was performed, and the experimental group, for which handgrip exercise and the 
same general physiotherapy were conducted. Throughout the experimental period, management of the area of the injury appropriate for the guidelines for the management following rotator cuff repair was implemented at the hospital ward for all subjects of this study. In addition, the patients received a doctor's prescription in accordance with the therapy guidelines after surgery and treatment by the resident doctor everyday throughout hospitalization. After discharge from the hospital, they underwent treatment and management of the injury by the resident doctor once every $3 \sim 7$ days.

To relax the muscles around the neck following the shoulder rotator cuff repair, occipital release was implemented by placing the palm underneath the back of the head of the subject, and gentle pressure was then applied from the area below the arch of the $1^{\text {st }}$ neck bone towards the nose of the subject using the tip of the finger of the experimenter for 15 minutes [14]. The area of injury was wrapped with a towel, and an ice pack was placed on top for the implementation of ice therapy for 15 minutes to reduce pain and edema. Moreover, a Sonopuls590 (ENRAF, Netherlands), an ultrasound therapeutic device, was used to induce tissue growth and regeneration of the surgical area. Ultrasound therapy was performed for five minutes by setting the frequency at $1 \mathrm{MHz}$, intensity at $3 \mathrm{~W} / \mathrm{cm}^{2}$ and irradiation format at a pulsed ultrasonic wave. The same general physiotherapy was applied to both the control group and experimental group six times per week for six weeks.

A dynamometer was used for the maximum power of grasp(1RM) to determine the intensity of the handgrip exercise for the subjects who underwent rotator cuff repair. Based on the measurement values, the intensity of the handgrip exercise was set to $50 \%$ of $1 \mathrm{RM}$. As instructed by the American society of hand therapists, the subjects were instructed to assume a comfortable seated posture with the shoulder joint adducted and turned in the neutral state. The elbow joint bent at $90^{\circ}$, and the wrist joint was in the neutral position before commencing the handgrip exercise. The subjects were asked to squeeze the dynamometer at $50 \%$ of the maximum grip power and maintain the hold for seven seconds, followed by relaxation for three seconds. This was set to one repetition with 10 repetitions composing a single set. The subjects underwent three sets of this handgrip exercise six times a week for six weeks in the physiotherapy room [15].

\section{Statistical analyses}

The results were analyzed using the SPSS for Windows 20.0 program. A normal distribution was assessed using a Kolmogorove-Smirnov test, and the linearity was explored through scatter plots. An independent sample t-test was performed to confirm the general characteristics of the research subjects and the homogeneity of the subjects prior to the experiment. Two-way repeated measure ANOVA was performed for the extent of activation of the muscles around the shoulder. To examine the cross-sectional area of the supraspinatus muscle, an independent sample t-test was conducted by calculating the variation in each group with the significance level(a) set to .05 .

\section{Results}

\section{General characteristics of the subjects}

This study examined 28 subjects composed of 14 in the experimental group and 14 in the control group. The average age of the subjects was $60.68 \pm 9.98$ years with an average height of $163.92 \pm 8.33 \mathrm{~cm}$, average weight of $63.89 \pm 10.20 \mathrm{~kg}$, and average BMI of $23.66 \pm 2.47 \mathrm{~kg} / \mathrm{m}^{2}$. No significant differences were observed between the experimental and control group, and accordingly, the two groups were found to be homogeneous. The experimental group was composed of eight males(57\%) and six females $(43 \%)$. Thirteen of the subjects had damage to the right side rotator cuff( $(93 \%)$, while one subject had damage to 
Table 1. General Characteristics of the Subjects

\begin{tabular}{|c|c|c|c|c|c|c|}
\hline & & & $\begin{array}{l}\text { Experimental Group } \\
\qquad(\mathrm{n}=14)\end{array}$ & $\begin{array}{l}\text { Control Group } \\
\quad(n=14)\end{array}$ & $\mathrm{t}$ & $\mathrm{p}$ \\
\hline \multicolumn{3}{|c|}{ Height $(\mathrm{cm})$} & $161.71 \pm 7.70$ & $166.14 \pm 8.63$ & -1.420 & .160 \\
\hline \multicolumn{3}{|c|}{ Weight (kg) } & $61.93 \pm 8.47$ & $65.86 \pm 11.67$ & -1.010 & .310 \\
\hline \multicolumn{3}{|c|}{ BMI $\left(\mathrm{kg} / \mathrm{m}^{2}\right)$} & $23.63 \pm 2.43$ & $23.69 \pm 2.60$ & -.060 & .950 \\
\hline \multirow{6}{*}{\multicolumn{2}{|c|}{ Muscle Activity (\%RVC) }} & Anterior Deltoid & $97.96 \pm 3.79$ & $97.45 \pm 4.24$ & .332 & .742 \\
\hline & & Middle Deltoid & $100.21 \pm 5.21$ & $101.43 \pm 4.03$ & -.690 & .496 \\
\hline & & Posterior Deltoid & $101.71 \pm 5.58$ & $99.64 \pm 4.75$ & 1.058 & .300 \\
\hline & & Pectoralis Major & $114.36 \pm 15.75$ & $114.57 \pm 16.48$ & -.035 & .972 \\
\hline & & Upper Trapezius & $134.79 \pm 20.12$ & $131.29 \pm 10.59$ & .576 & .570 \\
\hline & & Infraspinatus & $104.43 \pm 7.13$ & $110.79 \pm 9.18$ & -2.047 & .051 \\
\hline
\end{tabular}

Values are mean \pm standard deviation, RVC: Reference voluntary contraction

the left rotator cuff $(7 \%)$. Regarding the size of the damage, seven subjects had less than $1 \mathrm{~cm}$ damage $(50 \%)$, and seven subjects had damage sizes in the range of $1 \sim 3 \mathrm{~cm}(50 \%)$. The control group was composed of nine males(64\%) and five females(36\%). Thirteen of the subjects had damage to the right side rotator cuff $(93 \%)$, while one subject had damage to the left rotator cuff $(7 \%)$. Regarding the size of the damage, eight subjects had damage of less than $1 \mathrm{~cm}(57 \%)$, and six subjects had damage sizes in the range of $1 \sim 3 \mathrm{~cm}(43 \%)$. The two groups were deemed homogeneous because the $\% \mathrm{RVC}$ of the anterior deltoid, middle deltoid, posterior deltoid, pectoralis major, upper trapezius, and infraspinatus among the muscles around the damaged shoulder were similar in the experimental group and the control group(Table 1).

\section{Comparison of the changes in muscle} activation

A comparison of the difference in the activation of anterior deltoid, middle deltoid, posterior deltoid, and pectoralis major revealed no significant difference between the two groups. The activation of upper trapezius decreased significantly in the experimental group compared to the control, and the activation of the infraspinatus was significantly higher in the experimental group than the control $(\mathrm{p}<.05)$. The muscle activation of the two groups over time showed a significant decrease in the anterior deltoid and upper trapezius, and a significant increase in pectoralis major and infraspinatus $(\mathrm{p}<.05)$.

\section{Comparison of the changes in the} cross-sectional area of the supraspinatus muscle

An analysis of the variation in the value of the cross-sectional area of the supraspinatus muscle of the experimental group(pre: $558.25 \pm 157.17 \mathrm{~mm}^{2}$, post: $616.24 \pm$ $171.69 \mathrm{~mm}^{2}$ ) and control group(pre: $553.37 \pm 238.02 \mathrm{~mm}^{2}$, post: $\left.543.63 \pm 129.29 \mathrm{~mm}^{2}\right)$ revealed a significant difference $(\mathrm{p}<$ $.05)($ Table 3).

\section{Discussion}

A study on the rehabilitation protocol after a rotator cuff repair suggested that shoulder joint exercise and shoulder muscle activation should be restricted for at least four to 16 weeks when wearing an abduction sling. On the other hand, atrophy in the shoulder muscles may occur if the joint movement is limited by wearing a sling for 
Table 2. Change in Muscle Activity (\%RVC) of the Experimental and Control Groups

\begin{tabular}{|c|c|c|c|c|c|c|c|c|}
\hline \multirow{2}{*}{ M } & \multirow{2}{*}{ G } & \multirow{2}{*}{ pre } & \multirow{2}{*}{ post 2 weks } & \multirow{2}{*}{ post 4 weks } & \multirow{2}{*}{ post 6 weks } & $\mathrm{T}$ & $\mathrm{T} \times \mathrm{G}$ & G \\
\hline & & & & & & $\mathrm{F}$ & $\mathrm{F}$ & $\mathrm{F}$ \\
\hline \multirow{2}{*}{$\mathrm{AD}$} & EG & $97.96 \pm 3.79$ & $101.98 \pm 8.50$ & $95.00 \pm 6.32$ & $94.61 \pm 7.85$ & \multirow{2}{*}{$6.882^{*}$} & \multirow{2}{*}{1.783} & \multirow{2}{*}{676} \\
\hline & $\mathrm{CG}$ & $97.45 \pm 4.24$ & $97.86 \pm 5.26$ & $96.43 \pm 5.09$ & $94.67 \pm 7.72$ & & & \\
\hline \multirow{2}{*}{ MD } & EG & $100.21 \pm 5.21$ & $99.93 \pm 4.48$ & $99.50 \pm 2.71$ & $98.92 \pm 3.34$ & \multirow{2}{*}{.408} & \multirow{2}{*}{.042} & \multirow{2}{*}{.134} \\
\hline & $\mathrm{CG}$ & $101.43 \pm 4.03$ & $101.57 \pm 3.65$ & $100.43 \pm 4.86$ & $100.07 \pm 4.34$ & & & \\
\hline \multirow{2}{*}{ PD } & EG & $101.71 \pm 5.58$ & $103.36 \pm 5.03$ & $103.07 \pm 5.27$ & $105.93 \pm 5.82$ & \multirow{2}{*}{1.665} & \multirow{2}{*}{1.708} & \multirow{2}{*}{.110} \\
\hline & CG & $99.64 \pm 4.75$ & $102.14 \pm 4.24$ & $102.21 \pm 4.90$ & $101.00 \pm 8.60$ & & & \\
\hline \multirow{2}{*}{ PM } & EG & $114.36 \pm 15.75$ & $120.14 \pm 22.19$ & $126.43 \pm 22.71$ & $130.39 \pm 24.49$ & \multirow{2}{*}{$12.935^{*}$} & \multirow{2}{*}{.679} & \multirow{2}{*}{.740} \\
\hline & $\mathrm{CG}$ & $114.57 \pm 16.48$ & $124.64 \pm 21.18$ & $128.64 \pm 23.54$ & $133.79 \pm 24.52$ & & & \\
\hline \multirow{2}{*}{ UT } & EG & $134.79 \pm 20.12$ & $120.07 \pm 13.27$ & $114.21 \pm 15.20$ & $84.71 \pm 7.30$ & \multirow{2}{*}{$49.157^{*}$} & \multirow{2}{*}{$4.135^{*}$} & \multirow{2}{*}{.235} \\
\hline & CG & $131.29 \pm 10.59$ & $132.14 \pm 21.66$ & $107.14 \pm 13.77$ & $98.57 \pm 8.74$ & & & \\
\hline \multirow{2}{*}{$\operatorname{Inf}$} & EG & $104.43 \pm 7.13$ & $120.00 \pm 10.24$ & $129.29 \pm 10.48$ & $138.57 \pm 12.22$ & \multirow{2}{*}{$71.320^{*}$} & \multirow{2}{*}{$9.368^{*}$} & \multirow{2}{*}{.338} \\
\hline & CG & $110.79 \pm 9.18$ & $120.50 \pm 7.11$ & $123.57 \pm 7.74$ & $126.93 \pm 7.63$ & & & \\
\hline
\end{tabular}

Values are mean \pm standard deviation, ${ }^{*} \mathrm{p}<.05$, M: Muscle, G: Group, AD: Anterior deltoid, MD: Middle deltoid, PD: Posterior deltoid, PM: Pectoralis major, UT: Upper trapezius, Inf: Infraspinatus, EG: Experimental group, CG: Control group, T: time, T $\times$ G: Time $\times$ group

Table 3. Change in the Cross-sectional Area of the Supraspinatus

\begin{tabular}{ccccc}
\hline & Experimental Group $(\mathrm{n}=14)$ & Control Group $(\mathrm{n}=14)$ & $\mathrm{t}$ & $\mathrm{p}$ \\
\hline $\mathrm{CSA}\left(\mathrm{mm}^{2}\right)$ & $57.59 \pm 38.57$ & $-9.81 \pm 22.91$ & 3.364 & $.015^{*}$ \\
\hline
\end{tabular}

Values are mean \pm standard deviation, ${ }^{*} \mathrm{p}<.05$, CSA: Cross-sectional area

more than four weeks after the rotor cuff repair to prevent re-rupture [16]. In this study, the effects of handgrip exercise, which can be applied to patients who passed two weeks after shoulder rotator cuff repair, on the shoulder muscle activation, and the cross-sectional area of the supraspinatus muscle was analyzed to provide basic clinical data for early intervention after a rotator cuff repair.

The rotator cuff contributes to the dynamic stability of the shoulder joint so that the humerus head cannot escape the central axis of the scapula glenoid fossa while performing the various operations of the shoulder joint, including shoulder joint abduction, external rotation, and internal rotation [17]. Howell and Kraft [86] reported that shoulder joint anterior instability appears kinematically if supraspinatus and infraspinatus atrophy or weakness occurs. De Witte et al. [19] explained that the upward movement of the humerus head during shoulder joint abduction was due to hyperexcitability of the deltoid and upper trapezius to compensate for the weakness of the supraspinatus and infraspinatus caused by rotator cuff 
damage. Sigholm et al. [20] examined the electromyogram of six muscles around the shoulder according to shoulder the joint flexion angles with hand loads. When the hand load was applied, muscle activation increased more in infraspinatus among rotator cuffs, which are involved in shoulder stability, than shoulder joint flexion muscles, such as anterior deltoid.

In this study, there were significant differences in muscle activation of the anterior deltoid, pectoralis major, upper trapezius, and infraspinatus over time between the experimental and control groups. In particular, there was a significant difference in the upper trapezius and infraspinatus when the changes in muscle activation between the groups were compared. In the case of the upper trapezius, the value of muscle activation decreased over time, whereas that of infraspinatus increased, which corresponds to Sigholm et al. [20]. Sporrong et al. [21] reported that the maximum muscle activation of the deltoid decreased by $4-14 \%$ at maximum handgrip, which is a similar result to the present study. In addition, Hodder and Keir [22] reported a significant decrease in muscle activation of the anterior deltoid, trapezius, and latissimus dorsi as the handgrip strength increased. Similarly, in this study, the experimental group showed a $37 \%$ decrease in muscle activation of the upper trapezius after the experiment, whereas the control group showed a $24 \%$ reduction in muscle activation of upper trapezius after the experiment. This indicates a $13 \%$ larger reduction in the experimental group compared to the control group.

The major muscles of shoulder abduction are the upper trapezius and deltoid. On the other hand, shoulder impingement syndrome can occur if these two muscles alone are activated because the shoulder joint is a ball-and-socket joint, and the humerus head should be gliding together by rolling in scapula glenoid fossa. If that is not the case and only the humerus head is rolling, the humerus head can come out of the scapula glenoid fossa, and shoulder impingement syndrome appears at the top part of the shoulder joint. To prevent shoulder joint impingement syndrome, the humerus head should have a lower and posterior gliding together, and muscles, such as the rotator cuff, lower trapezius, and anterior serratus muscles, should be activated to make such movements [23]. The supraspinatus muscle assists in the abduction movement of the shoulder and provides dynamic stability by pulling the humerus head into the scapula glenoid fossa during shoulder joint abduction so that the humerus head does not escape the scapula glenoid fossa.

If supraspinatus fails to function, the hyperstimulation of the shoulder joint abduction muscles in the upper trapezius and deltoid replaces the function of the supraspinatus muscle. Abduction of the shoulder joint due to such hyperstimulation of the deltoid and upper trapezius causes upward gliding of the humerus head in the scapula glenoid fossa, resulting in shoulder joint impingement syndrome [24]. A significant increase in the supraspinatus cross sectional area and a decrease in muscle activation of the upper trapezius and anterior deltoid due to early handgrip exercise after rotator cuff repair can prevent these shoulder joint imbalances and impart dynamic stability to the shoulder joint. This will allow bending and abduction of the shoulder joint without the humerus head escaping the scapula glenoid fossa.

In addition, the occurrence of shoulder impingement syndrome can be caused by the infraspinatus not compensating for deltoid hyperstimulation, resulting in the humerus head deviating from the scapula glenoid fossa. Weakening of the infraspinatus causes hyperstimulation of the deltoid, causing the humerus head to show upward gliding in the scapula glenoid fossa [25]. Therefore, a decrease in muscle activation of the anterior deltoid and the significant increase in muscle activation of infraspinatus by handgrip exercise in this study are expected to prevent rotator cuff rupture due to the shoulder joint impingement syndrome.

One of the main causes of supraspinatus atrophy is rotor 
cuff rupture [26]. The existence of rotator cuff rupture and the size of rupture are not only important factors in determining the treatment method but are also associated with the rehabilitation result. In many studies, atrophy of the supraspinatus was one of the most important prognostic factors after a rotator cuff repair [27-29]. MRI is generally used to determine the existence and size of rotator cuff rupture as well as the degree of atrophy [30]. Thomazeau et al. [13] used MRI to measure the cross-sectional area of the supraspinatus of patients with rotator cuff damage and normal subjects at the Y-shaped position, which is formed by the ends of the scapula bone, coracoid process, and clavicle, in the same way as this experiment. They reported that the supraspinatus cross-sectional area of patients with rotator cuff damage decreased by $30 \%$.

The results of this study showed that the supraspinatus cross-sectional area of the experimental group increased $18 \%$ after the handgrip exercise compared to that before the exercise, but the supraspinatus cross-sectional area of the control group decreased by $3 \%$. Chung et al. [31] measured the supraspinatus atrophy by MRI before and one year after surgery in 191 patients with a rotator cuff injury. The measurement method of supraspinatus atrophy was the same as in the present study, and the area of the supraspinatus after successful arthroscopic repair increased by $17 \%$. In this study, the cross-sectional area of the supraspinatus was increased by $18 \%$ with handgrip exercise, which was performed two to six weeks after the rotator cuff repair. This indicates that handgrip exercise is an excellent way to increase the supraspinatus cross-sectional area.

On the other hand, the effects of general physical therapy and handgrip exercise combined with general physical therapy were compared, and the handgrip exercise was performed with caution during the additional time given to the patients. To clarify which intervention is more effective, the effects of the handgrip exercise should be assessed alone in future studies.

\section{Conclusion}

Early handgrip exercise after rotator cuff repair increased the muscle activation of the infraspinatus and the cross-sectional area of the supraspinatus. Because the infraspinatus and supraspinatus muscles are important for the dynamic stability of the shoulder joint, handgrip exercise will be an effective way to activate the muscles around the shoulder joint when the shoulder joint movement is restricted to prevent re-rupture after rotator cuff repair.

\section{References}

[1] McBeth J, Jones K. Epidemiology of chronic musculoskeletal pain. Best Pact Res Clin Rheumatol. 2007;21(3):403-25.

[2] Meyer DC, Gerber C. Failure of anterior shoulder instability repair caused by eyelet cutout of absorbable suture anchors. Arthroscopy. 2004;20(5):521-3.

[3] Hong F, Haldeman BD, John OA, et al. Characterization of tightly associated smooth muscle myosin-myosin light-chain kinase-calmodulin complexes. J Mol Biol. 2009;390(5):879-92.

[4] Bartolozzi A, Andreychik D, Ahmad S. Determinants of outcome in the treatment of rotator cuff disease. Clin Orthop Relat Res. 1994;308:90-7.

[5] Green A. Chronic massive rotator cuff tears: evaluation and management. J Am Acad Orthop Surg. 2003;11(5): 321-31.

[6] Boudreau S, Boudreau ED, Higgins LD, et al. Rehabilitation following reverse total shoulder arthroplasty. J Orthop Sport Phys Ther. 2007;37(12):734-43.

[7] Koo SS, Burkhart SS. Rehabilitation following arthroscopic rotator cuff repair. Clin Sports Med. 2010;29(2):203-11.

[8] Psatha M, Wu Z, Gammie FM, et al. A longitudinal MRI study of muscle atrophy during lower leg immobilization following ankle fracture. J Magn Reson Imaging. 2012;35(3):686-95.

[9] Kibler WB, Press J, Sciascia A. The role of core stability 
in athletic function. Sports Med. 2006;36(3):189-98.

[10] Au AK, Keir PJ. Interfering effects of multitasking on muscle activity in the upper extremity. J Electromyogr Kinesiol. 2007;17(5):578-86.

[11] DeOrio JK, Cofield RH. Results of a second attempt at surgical repair of a failed initial rotator-cuff repair. J Bone Joint Surg Am. 1984;66(4):563-7.

[12] Cram JR, Kasman GS, Holtz J. Introduction to Surface Electromyography. Gaithersburg, MD: Aspen Publishers, Inc. 1998.

[13] Thomazeau H, Rolland Y, Lucas C, et al. Atrophy of the supraspinatus belly. Assessment by MRI in 55 patients with rotator cuff pathology. Acta orthop Scand. 1996;67(3):264-8.

[14] Manheim CJ, Lavett DK. The myofascial release manual. Thorofare(NJ): Slack. 1994.

[15] American college of sports medicine. Guidelines for exercise testing and prescription. 8th ed. Philadelphia (PA): Lippincott, Wilkins, and Williams. 2009.

[16] Ross D, Maerz T, Lynch J, et al. Rehabilitation following arthroscopic rotator cuff repair; a review of current literature. J Am Acad Orthop Surg. 2014;22(1):1-9.

[17] Escamilla RF, Yamashiro K, Paulos L, et al. Shoulder muscle activity and function in common shoulder rehabilitation exercises. Sports Med. 2009;39(8):663-85.

[18] Howell SM, Kraft TA. The role of the supraspinatus and infraspinatus muscles in glenohumeral kinematics of anterior should instability. Clin Orthop Relat Res. 1991;(263):128-34.

[19] De Witte PB, van der Zwaal P, Visch W, et al. Arm adductor with arm abduction in rotator cuff tear patients vs. healthy design of a new measuring instrument. Hum Mov Sci. 2012;31(2):461-71.

[20] Sigholm G, Herbets P, Almstrom C, et al. Electromyographic analysis of shoulder muscle load. J Orthop Res. 1984; 1(4):379-86.
[21] Sporrong H, Palmerud G, Herberts P. Influences of handgrip on shoulder muscle activity. Eur J Appl Physiol Occup Physiol. 1995;71(6):485-92.

[22] Hodder JN, Keir PJ. Targeted gripping reduces shoulder muscle activity and variability. J Electromyogr Kinesiol. 2012;22(2):186-90.

[23] Koester MC, George MS, Kuhn JE. Shoulder impingement syndrome. Am J Med. 2005;118(5):452-5.

[24] Reddy AS, Mohr KJ, Pink MM, et al. Electromyographic analysis of the deltoid and rotator cuff muscles in persons with subacromial impingement. J Shoulder Elbow Surg. 2000:9(6):519-23

[25] Clisby EF, Bitter NL, Sandow MJ, et al. Relative contributions of the infraspinatus and deltoid during external rotation in patients with symptomatic subacromial impingement. J Shoulder Elbow Surg. 2008;17(1):87-92.

[26] Oh JH, Kim SH, Ji HM, et al. Prognostic factors affecting anatomic outcome of rotator cuff repair and correlation with functional outcome. Arthroscopy. 2009;25(1):30-9.

[27] Gladstone JN, Bishop JY, Lo IK, et al. Fatty infiltration and atrophy of the rotator cuff do not improve after rotator cuff repair and correlate with poor functional outcome. Am J Sports Med. 2007;35(5):719-28.

[28] Goutallier D, Postel JM, Gleyze P, et al. Influence of cuff muscle fatty degeneration on anatomic and functional outcomes after simple suture of full-thickness tears. J Shoulder Elbow Surg. 2003;12(6):550-4.

[29] Liem D, Lichtenberg S, Magosch P, et al. Magnetic resonance imaging of arthroscopic supraspinatus tendon repair. J Bone Joint Surg Am. 2007;89(8):1770-6.

[30] Khoury V, Cardinal E, Brassard P. Atrophy and fatty infiltration of the supraspinatus muscle; sonography versus MRI. AJR Am J Roentgenol. 2008;190(4):1105-11.

[31] Chung SW, Kim SH, Tae SK, et al. Is the supraspinatus muscle atrophy truly irreversible after surgical repair of rotator cuff tears? Clin Orthop Surg. 2013;5(1):55-65. 